\title{
Assessment of adherence to anti tuberculosis medication for successful implementation of revised national tuberculosis programme at a tertiary care hospital, Shimoga: a cross-sectional observational study
}

\author{
Vedavathi Hanumaiah ${ }^{1}$, Dharani Devangi Ranganath ${ }^{1}$, Nataraja Kakkuppi ${ }^{2}$
}

\begin{abstract}
${ }^{1}$ Department of Pharmacology, Shimoga Institute of Medical Sciences, Shimoga, Karnataka, India

${ }^{2}$ District TB centre, Shimoga, Karnataka, India
\end{abstract}

Received: 25 September 2019

Revised: 11 October 2019

Accepted: 12 October 2019

\section{*Correspondence to:}

Dr. Dharani Devangi Ranganath, Email: dharanidevangi2707@ gmail.com

Copyright: (C) the author(s), publisher and licensee Medip Academy. This is an openaccess article distributed under the terms of the Creative Commons Attribution NonCommercial License, which permits unrestricted noncommercial use, distribution, and reproduction in any medium, provided the original work is properly cited.

\begin{abstract}
Background: This study assessed level of non-adherence to anti tuberculosis (TB) therapy among pulmonary TB patients, compares various factors among adherent and non-adherent TB patients, stressing on reasons for non-adherence at a tertiary care hospital.

Methods: This institution based observational and cross-sectional study was conducted interviewing patients with pulmonary TB and assessed using Moriskys medication adherence scale-8 (MMAS-8), a pre- tested structured questionnaire based scoring system of patients treated for pulmonary $\mathrm{TB}$ at district TB centre SIMS, Shimoga. Descriptive statistics were employed.

Results: Among 70 cases analysed, 57 were males and 13 females, with mean age group of $41.32 \pm 8.63$ and mean MMAS score of $2.23 \pm 1.87$. 53.33\% patients were on continuous phase of treatment. The level of non-adherence were as follows, high $=18 \%$, medium $=38 \%$ and low $=44 \%$. The common cause for nonadherence was forgetfulness $(66 \%)$ reasons being: betterment of symptoms $(54 \%)$, sickness after taking medication $(31 \%)$, distance of travel: far $(15 \%)$. Many were labourers $(62 \%)$, with low literacy rate, also chronic alcoholics $(72 \%)$ and smokers $(73 \%)$. Female with moderate literacy and not addicted to alcohol/smoking showed high adherence compared to males $(\mathrm{p}<0.05 \%)$.

Conclusions: As prevalence of non-adherence is high, especially Patients on continuous phase of TB treatment, there arises immense need for continuous and effective health education to patients' and their family regarding the adverse effects and the need for high level of adherence to treatment for the complete cure of disease. Patients who are addicted to alcohol/smoking should be targeted with interventions to quit the same, provide free transport facility to RNTCP centres and prompt treatment of ADR, will improve adherence to medication.
\end{abstract}

Keywords: Adherence, Anti-tuberculosis treatment, Non-adherence, Pulmonary TB

\section{INTRODUCTION}

Tuberculosis (TB) is one of the oldest diseases known to human and is a major cause of death worldwide. About $1 / 3^{\text {rd }}$ of the world's population is estimated to be infected with tubercle bacilli and hence at risk of developing active disease. TB is a major contributor to the global burden of disease, particularly in low and middle income countries due to its association with human immunodeficiency virus. Lack of adherence to anti-TB therapy is a major factor for the re-emergence of $\mathrm{TB}^{1-3} \mathrm{~TB}$ treatment presents particular challenges for adherence because the treatment is long and involves taking a number of medications, side-effects are common and the patient usually feels better long before treatment has been completed. Non-adherence has been cited as one of the reasons for failure of achievement of the global treatment success rates throughout the world. ${ }^{4}$ In order to successfully control TB there exists a requirement that we completely understand all the factors involved in a patient's ability to adhere to treatment. Non-adherence to anti-TB therapy has been a persistent problem throughout the world, including both developing and developed nations. Several studies from developed or industrialised 
nations have shown that alcohol usage, low literacy level, low annual income, younger age, and longer duration of treatment were associated with nonadherence, whereas adherence was higher among patients with TB who were initially hospitalized and those who returned for followup within 4 weeks of initiation of therapy. ${ }^{5-7}$

In developing countries, adherence to TB therapy seems to be related to shorter home-to-clinic distances. ${ }^{8,9}$ The use of short-course therapy, whether or not the patient returns for a repeat smear, and whether the patient is reevaluated in the same unit and district. ${ }^{8}$ Poverty, difficulty supporting the family, the fear of injections, the fear of having to ask for financial support from employers, the fear of loss of employment, and the fostering of healthdamaging beliefs were all associated with non-adherence to TB therapy in developing countries. ${ }^{10}$

Non-adherence to TB treatment leads to delayed sputum conversion, higher relapse rates and emerging drug resistance. $^{11}$

In addition, the emergence and rapid growth of multidrug resistant (MDR) TB has become a matter of great concern. $^{12,13}$

Patients with TB are expected to have adherence levels greater than $90 \%$ in order to facilitate cure. Failure for cure increases the risk of development of drug resistant strains, spread of TB in the community and this in turn increases morbidity and mortality. The burden of TB in Uganda is already very high with the country ranking 15th among the high burden countries. ${ }^{14}$ Any further increases in the burden of TB would overstretch the health system that is already over laden with TB patients. The problem would be worse with the multi-drug (MDR) and extensively drug resistance (XDR) TB cases that are likely to develop with non-adherence and are difficult to treat. $^{15}$

Studies that have been done elsewhere have found the prevalence of non-adherence of $21.2-32.9 \% .^{16,17} \mathrm{~A}$ number of factors associated with non-adherence have been cited in some of the literature which includes health system, condition related, patient related, socioeconomic and therapy related factors. ${ }^{18}$

The concept of directly observed treatment short course (DOTS) in TB was developed in an attempt to enhance patient adherence to chemotherapy, which is crucial for the successful management of the disease at both the individual and community levels. Although DOT was introduced in Turkey in 2002, the coverage rate of DOT is still less than $10 \%$ of the total population. ${ }^{19}$

The objective of the study is to assess the level of nonadherence to anti TB therapy among patients with pulmonary $\mathrm{TB}$ and compares various factors among adherent and non-adherent TB patients, stressing on reasons for non-adherence at a tertiary care hospital.

\section{METHODS}

A prospective, cross-sectional, observational medical audit was conducted for a period of 6 weeks (June 2017July 2017) at district TB centre, Mc Gann Teaching Hospital, Shimoga.

All patients suffering from pulmonary tuberculosis attending ATT centre under the institution were counselled in order to obtain continuity in follow up, also explaining the need for adherence to treatment for complete cure. Quantitative data was collected by trained research assistants using a pre-tested, semi-structured questionnaire. The questionnaire was translated to the area's local language (Kannada) and back translated to English to ensure consistency of meaning. This institution based observational and cross-sectional review was conducted interviewing patients with Pulmonary TB and assessed using Moriskys medication adherence scale- 8 (MMAS-8), a pre- tested structured questionnaire based scoring system of patients treated for pulmonary TB. Inclusion criteria were newly diagnosed pulmonary $\mathrm{TB}$ patients of either gender who were on anti TB follow up for the last one month with age above 18 years. Exclusion criteria were patients with treatment relapse or failure, extra pulmonary TB. The approval from Institutional Ethics Committee was obtained accordingly. Data was also collected on patient socio demographic details and program factors e.g. distance to the treatment centre, availability of drugs and cost of travel to the centre.

- Socio demographic data: Name, age, sex, address, nature of work (employment), literacy levels, marital status, mode of transportation to hospital, time to reach hospital from residence.

- Medical history: Diagnosis, treatment status, availability of drugs, frequency of administration, route of administration, any side effects or adverse effects reported smoking and alcoholics.

- Data pertaining to level of adherence assessment: As per Morisky-8 item medication adherence scale (Table 1).

Table 1: Morisky-8 item medication adherence scale.

\begin{tabular}{|ll|}
\hline Do you sometimes forget to take your medicines? & No $=1, \mathrm{yes}=0$ \\
\hline Over past two weeks were tere any days you forgot to take your medicines? & $\mathrm{No}=1, \mathrm{yes}=0$ \\
\hline $\begin{array}{l}\text { Have you ever stopped taking medicine without telling your doctor as you started feeling } \\
\text { worse with the medicines? }\end{array}$ & $\mathrm{No}=1, \mathrm{yes}=0$ \\
\hline When you travel or leave home do you forget to take medicines with you? & $\mathrm{No}=1, \mathrm{yes}=0$ \\
\hline Did you take all your medicines yesterday? & Yes $=1, \mathrm{no}=0$ \\
\hline
\end{tabular}

Continued. 


\begin{tabular}{|l|l|}
\hline $\begin{array}{l}\text { When you feel like your symptoms are under control, do you feel like stop taking } \\
\text { medicines? }\end{array}$ & No=1, yes=0 \\
\hline Do you ever feel hassled about sticking to your treatment plan? & No=1, yes $=0$ \\
\hline How often do you have difficulty to remember taking you medicines? & \\
\hline A) Never/rarely & $\mathrm{A}=4$ \\
\hline B) Once in a while & $\mathrm{B}=3$ \\
\hline C) Sometimes & $\mathrm{C}=2$ \\
\hline D) Usually & $\mathrm{D}=1$ \\
\hline E) All the time & $\mathrm{E}=0$ \\
\hline & Divide total score by 4 \\
\hline
\end{tabular}

Score $<6=$ low adherence; score $6-8=$ medium adherence; score $8=$ high adherence.

\section{Statistical analysis}

Descriptive statistical analysis was done using Microsoft Office Excel 2013 and comparative analysis of data was performed using chi-square test by SPSS software version 19.

\section{RESULTS}

A total of 70 cases were interviewed and analysed. The mean age of patients undergoing treatment for pulmonary TB was found to be $41.32 \pm 1.63$. Among them were female patients constituting $13(18.57 \%)$ and male patients $57(81.42 \%)$. About $53.33 \%$ patients were on continuous phase of treatment. Mean MMAS score of $2.23 \pm 1.87$, the level of non-adherence were as follows, high $=18 \%$, medium $=38 \%$ and low $=44 \%$ (Figure 1$)$. The most common cause for non-adherence was forgetfulness (66\%) (Figure 2) and others causes constituted to about $44 \%$ of those causes most patients voluntarily stopped taking medicines without consulting their doctors since they felt better or relieved from symptoms (Figure 3). Travel distance from patient's residence to nearby TB centre was recorded and $48 \%$ of patients seemed to have been residing more than $6 \mathrm{~km}$ far from TB centre (Table 2).

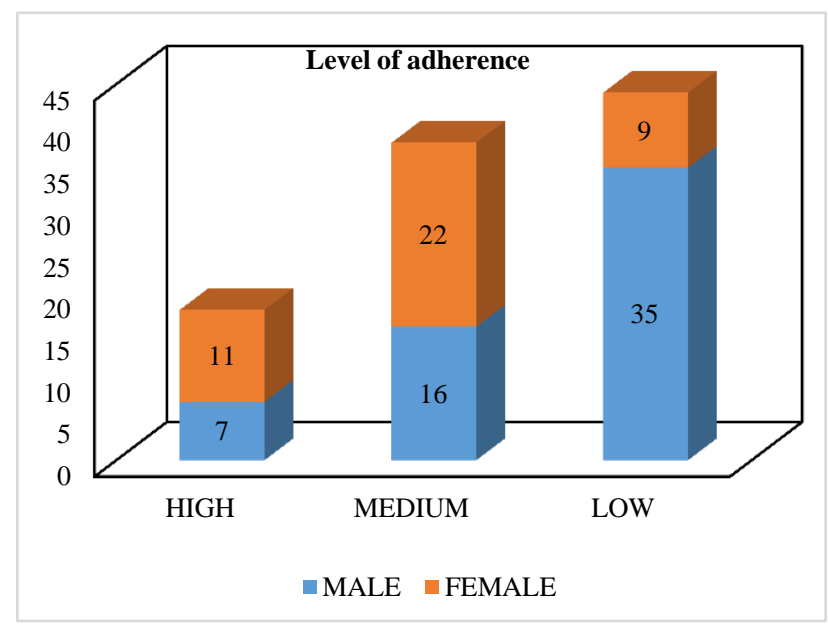

Figure 1: Level of adherence to treatment among TB patients.

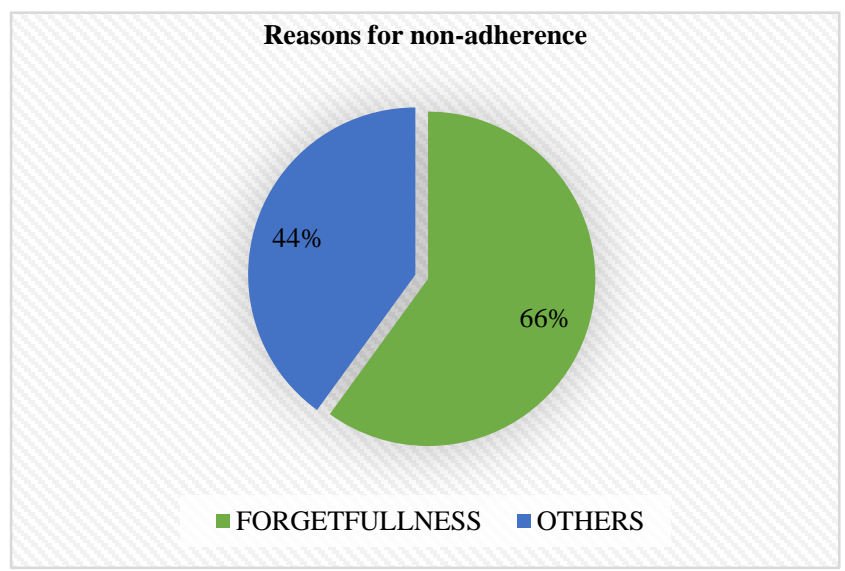

Figure 2: Reasons for non-adherence among TB patients.

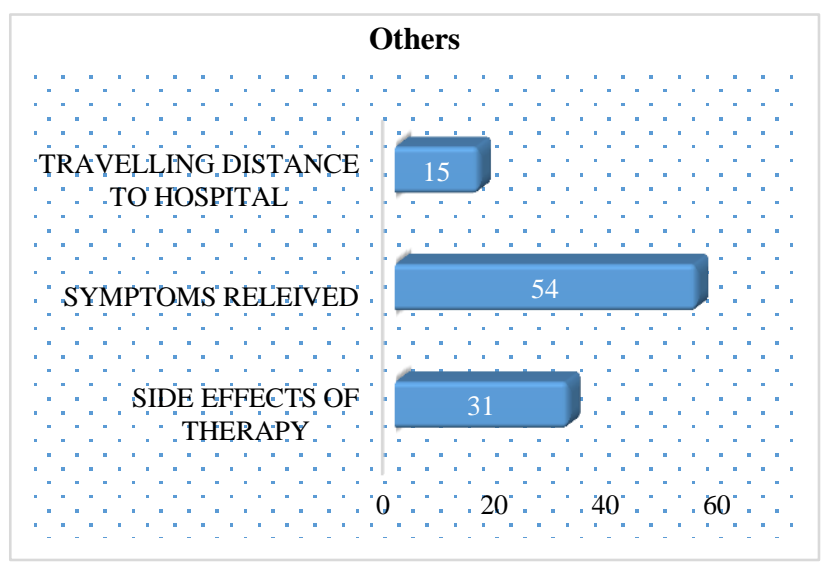

Figure 3: Other reasons for non-adherence among TB patients.

Table 2: Travel distance to reach district TB centre.

\begin{tabular}{|l|l|}
\hline \multicolumn{1}{|c|}{ Far $>\mathbf{6} \mathbf{~ k m}$} & Percentage (\%) \\
\hline Short $\mathbf{2 - 6} \mathbf{~ k m}$ & 48 \\
\hline Nearer $<\mathbf{2}$ km & 32 \\
\hline
\end{tabular}

Common side effects observed during the treatment were that most people experienced dizziness, urine discolouration and skin rashes. Also few patients 
experienced headache, vomiting and yellowish discolouration of eye (Figure 4).

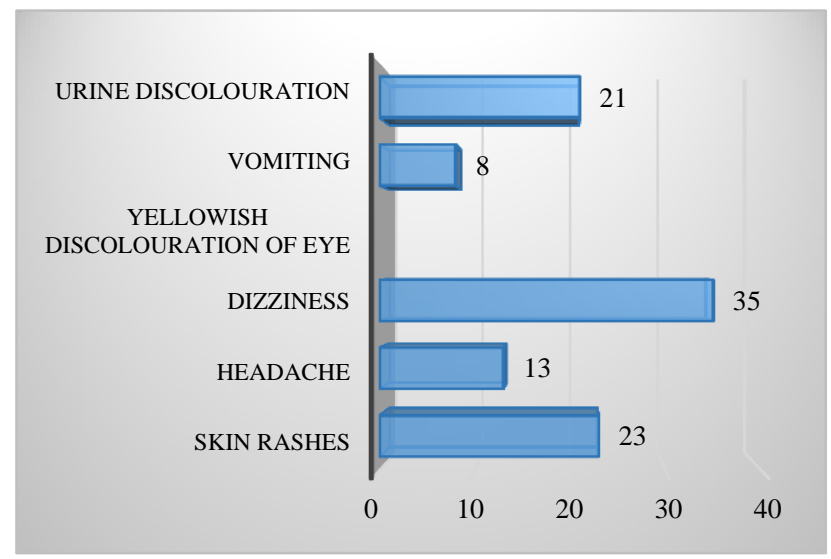

Figure 4: Observed side effects during treatment.

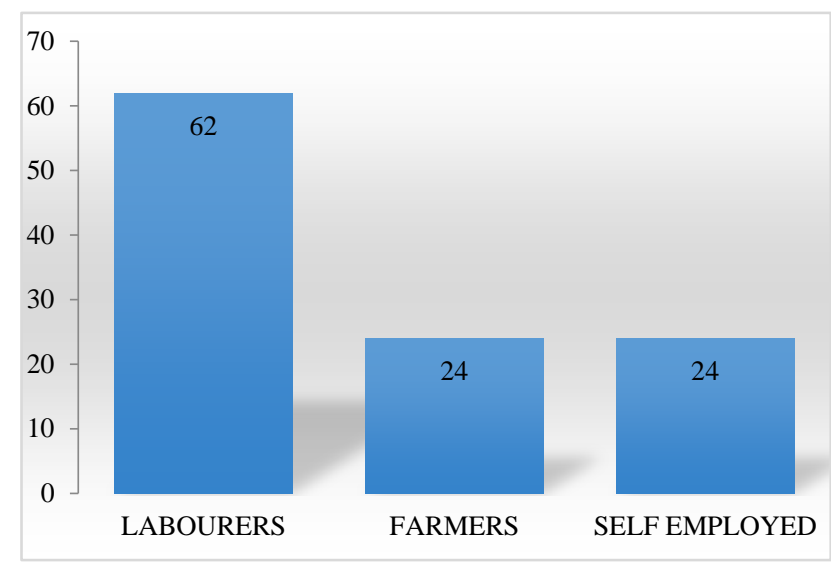

Figure 5: Nature of employment.

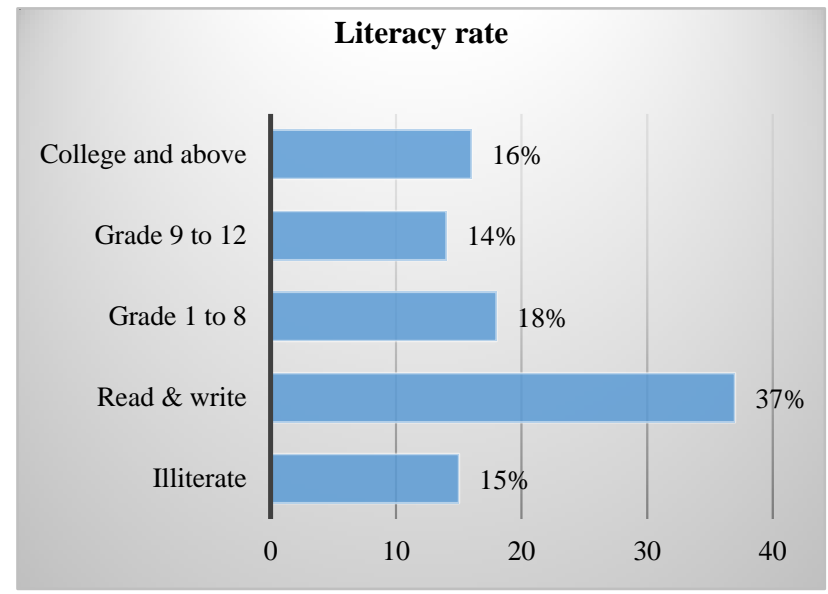

Figure 6: Literacy rate among TB patients.

Nature of employment and patient's literacy rate were also assessed. $62 \%$ of patients were labourers working on daily wages and rest were farmers and self-employed (Figure 5), also $37 \%$ of patients could only read and write, very few had completed schooling and graduation (Figure 6). Association between habits of abuse and level of adherence were analysed and it was found that very low adherence was seen among alcoholics and smokers with significant $\mathrm{p}<0.0032$ (Figure 7).

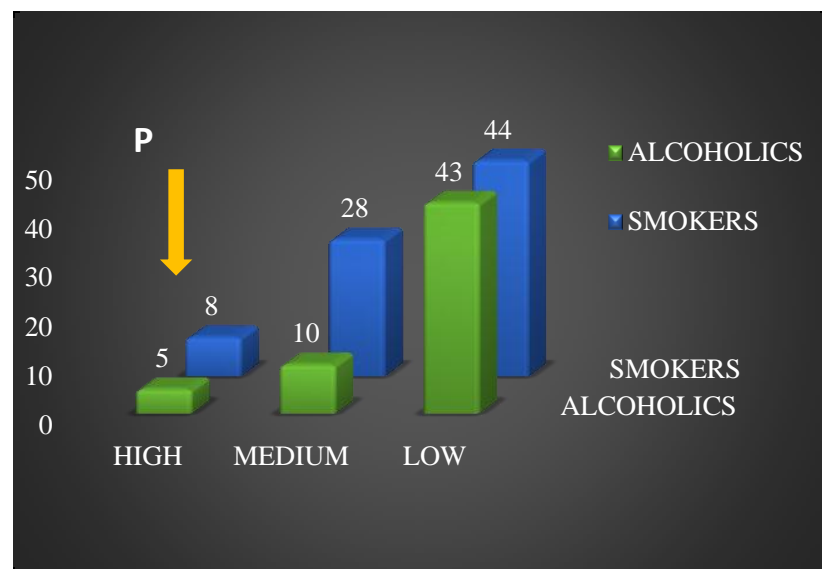

Figure 7: Association between habits of abuse and level of adherence.

\section{DISCUSSION}

Adherence to anti-TB treatment is a major determinant of treatment outcome. In developing countries where inequities in access to health care are high and health resources are scarce, the magnitude and impact of poor adherence is assumed to be higher. Hence, this study assessed the level and describes factors of non-adherence to anti-TB medications. About $66 \%$ of non-adherence was due to forgetfulness of patients to go to DOTS clinic and take medicine. Such ignorance on part of patients reflects inadequate dissemination of information and education regarding TB and importance of its treatment by DOTS providers. Other more than $15 \%$ of non-adherence was due to far distance from their residence to the DOT centres. Also these factors are indirectly related to cost of treatment.

Among newly diagnosed patient's it is a challenge to balance the routine and hold on to the commitment to be adherent. Thus, it appears that provision of free treatment alone is not sufficient to facilitate adherence. Findings of this study was more than twice $(19.2 \%)$ that of similar study done in Kolkata, but these findings are consistent with the results reported from studies conducted in other developing countries. Lack of family and social support predicts poor treatment adherence. ${ }^{20}$ Alcohol consumption is well known risk factor for non-adherence. ${ }^{21}$ Finding of this study showed $42(60 \%)$ alcoholics in our study. This study is slightly higher than (33.3\%) study in Uganda. As, smoking is most of the times associated with alcoholism, it can also predict poor treatment adherence. ${ }^{22}$ Out of 70 patients, in this study, there were $43(61.42 \%)$ smokers. Even though there is no consensus among the studies investigating gender-associated adherence to TB treatment, it is hypothesized that one explanation for studies showing women to be more compliant with TB treatment is that barriers to diagnosis of $\mathrm{TB}$ in women 
screen out those women most likely to default from treatment. $^{23,24}$ Those who overcome diagnostic barriers are highly motivated and are thus most likely to comply with treatment. Barriers to adherence especially relevant for women include lack of time, cash, transportation, and replacement labour, whereas alcohol consumption plays an important role as a barrier to adherence in men. Nonpregnant women are believed to be more adherent than men. $^{25}$

\section{CONCLUSION}

Female patients with moderate literacy level and not addicted to alcohol/smoking showed high adherence compared to males $(\mathrm{p}<0.05 \%)$. Under the DOTS strategy, patients are expected to attend a TB treatment facility every day, at least for the first 2 months of the intensive phase, for direct observation of treatment, therefore accruing indirect costs due to lost income. If patients attended less frequently to collect their drugs, it would be more convenient for both patients and health providers. Procedures that supervise treatment closer to the patient's residence would be even more convenient, and more importantly, would spare patients the burden of losing income from work. This is particularly true in a developing country like India where, for the majority of population, daily earnings are crucial to survival.

As prevalence of non-adherence is high, especially those Patients who are on continuous phase of TB treatment, there arises immense need for continuous and effective health education to patients' and their family regarding the adverse effects and the need for high level of adherence to treatment for the complete cure of disease. In addition, patients who are addicted to alcohol \& smoking should be targeted with interventions to quit the same, also provide free transport facility to RNTCP/SIMS centres and prompt treatment of adverse effects of drugs, will improve adherence to medication.

\section{ACKNOWLEDGEMENTS}

We thank our district TB centre staffs, our colleagues and resident post graduate students for their continued support during this study.

Funding: No funding sources Conflict of interest: None declared

Ethical approval: The study was approved by the Institutional Ethics Committee

\section{REFERENCES}

1. Barnhoorn F, Adriaanse H. In search of Factors responsible for noncompliance among tuberculosis Patients in Wardha District, India. Soc Sci Med. 1992;34:291-306.

2. Williams G. Holding the patient. Ann N Y Acad Sci. 2001;953:199-207.
3. Johansson E, Diwan VK, Huong ND, Ahlberg BM. Staff and patient attitudes to tuberculosis And compliance with treatment:an exploratory study in A district in Vietnam. Tuber Lung Dis. 1996;77:178-83.

4. WHO. Global tuberculosis report: Uganda. WHO report. 2007: 145-147.

5. McDonnell M, Turner J, Weaver MT. Antecedents of adherence to antituberculosis therapy. Public Health Nurs. 2001;18:392-400.

6. Ormerod LP, Prescott RJ. Inter-relations between relapses, drug regimens and compliance with treatment in tuberculosis. Respir Med. 1991;85:23942.

7. Menzies R, Rocher I, Vissandjee B. Factors associated with compliance in treatment of tuberculosis. Tuber Lung Dis. 1993;74:32-7.

8. Erhabor GE, Aghanwa HS, Yusuph M, Adebayo RA, Arogundade FA, Omidiora A. Factors influencing compliance in patients with tuberculosis on directly observed therapy at Ile-Ife, Nigeria. East Afr Med J. 2000;77:235-9.

9. Van Der Werf TS, Dade GK, Van Der Mark TW. Patient compliance with tuberculosis treatment in Ghana: factors influencing adherence to therapy in a rural service programme. Tubercle. 1990;71:247- 52.

10. Nuwaha F. Factors influencing completion of treatment among tuberculosis patients in Mbarara District, Uganda. East Afr Med J. 1990;74:690-3.

11. World Health Organization, Global Tuberculosis Control. Surveillance, Planning, Financing. 170, Geneva, 2004.

12. Tahao glu K, Törün T, Sevim T, Atac G, Kir A, Karasulu L, Kapakli N. The treatment of multidrugresistant tuberculosis in Turkey. $\mathrm{N}$ Engl $\mathrm{J}$ Med. 2001;345:170-4.

13. Kilicaslan Z, Ozturk F, Sarimurat N, Cuhadaroglu C, Caglar E, Erem A. Microscopic examination and treatment outcomes of new pulmonary tuberculosis cases in Istanbul dispensaries between 1998 and 2000. Int J Tuberc Lung Dis. 2003;7:1059-63.

14. WHO. Global tuberculosis report:Uganda. WHO report. 2007: 145-147.

15. Awofeso N. Antituberculous medication side-effects constitute major factor for poor adherence to tuberculosis treatment. Bulletin of WHO. 2008: 86.

16. Rocha M, Pereira S, Ferreira L, Barros H. The role of adherence in TB-HIV positive patients in ambulatory regimen. Euro Resp J. 2003;21:785-8.

17. Meissner PE, Musoke P, Okwira A, Bunn JEG, Coutter JBS. The value of urine testing for verifying adherence to anti-TB chemotherapy in children and adults in Uganda. Int $\mathbf{J}$ Tuberculosis Lung Dis. 2002;6(10):903-8.

18. Tuberculosis coalition for technical assistance. International standards for Tuberculosis care. In $\mathrm{j}$ tuberculosis and lung diseases. 2006;56:198-201.

19. World Health Organization): Treatment of tuberculosis: guidelines for national programmes. 2003: 21-26. 
20. Kumareson J. Tuberculosis: Epidemiology and Control. 1st Ed. New Delhi: WHO Regional Office for South-East Asia. 2002: 16-17.

21. World Health Organization, WHO Report 2011 Global Tuberculosis Control. France WHO. 2011;258.

22. Sophia V, Balasangameshwara VH, Jagannatha PS, Saroja VN, Shivashankar B, Jagota P. Retreatment outcome of smear positive PTB patients under DOTS in Bangalore city. Indian $\mathrm{J}$ Tuberculosis. 2002;49:195-203.

23. Connolly M, Nunn P. Women and tuberculosis. World Health Stat Is. 1996;49:115-9.

24. Nichter N. Illness semantics and international health: the weak lungs/TB complex in the Philippines. Soc Sci Med. 1994;38:649-63.
25. Long NH, Johansson E, Diwan VK, Winkvist A. Fear and social isolation as consequences of 158 tuberculosis in Vietnam: a gender analysis. Health Policy. 2001;58:69-81.

Cite this article as: Hanumaiah V, Ranganath DD, Kakkuppi N. Assessment of adherence to anti tuberculosis medication for successful implementation of revised national tuberculosis programme at a tertiary care hospital, Shimoga: a cross-sectional observational study. Int J Basic Clin Pharmacol 2019;8:2361-6. 